\title{
Molecular docking analysis of S-alkyl derivatives of thiosalicylic acid as cyclooxygenase inhibitor agents
}

Miloš V. Nikolića

Aleksandar Kočovića , Verica V. Jevtićć, Zoran R. Ratkovićc, Srećko R. Trifunovićć, Gordana P. Radića

${ }^{a}$ University of Kragujevac, Faculty of Medical Sciences, Department of Pharmacy, Svetozara Markovića 69, 34000 Kragujevac, Serbia

${ }^{b}$ University of Belgrade, Faculty of Pharmacy, Department of Pharmaceutical Chemistry, Vojvode Stepe 450, 11000 Belgrade, Serbia

cUniversity of Kragujevac, Faculty of Science, Department of Chemistry, Radoje Domanovića 12, 34000 Kragujevac, Serbia

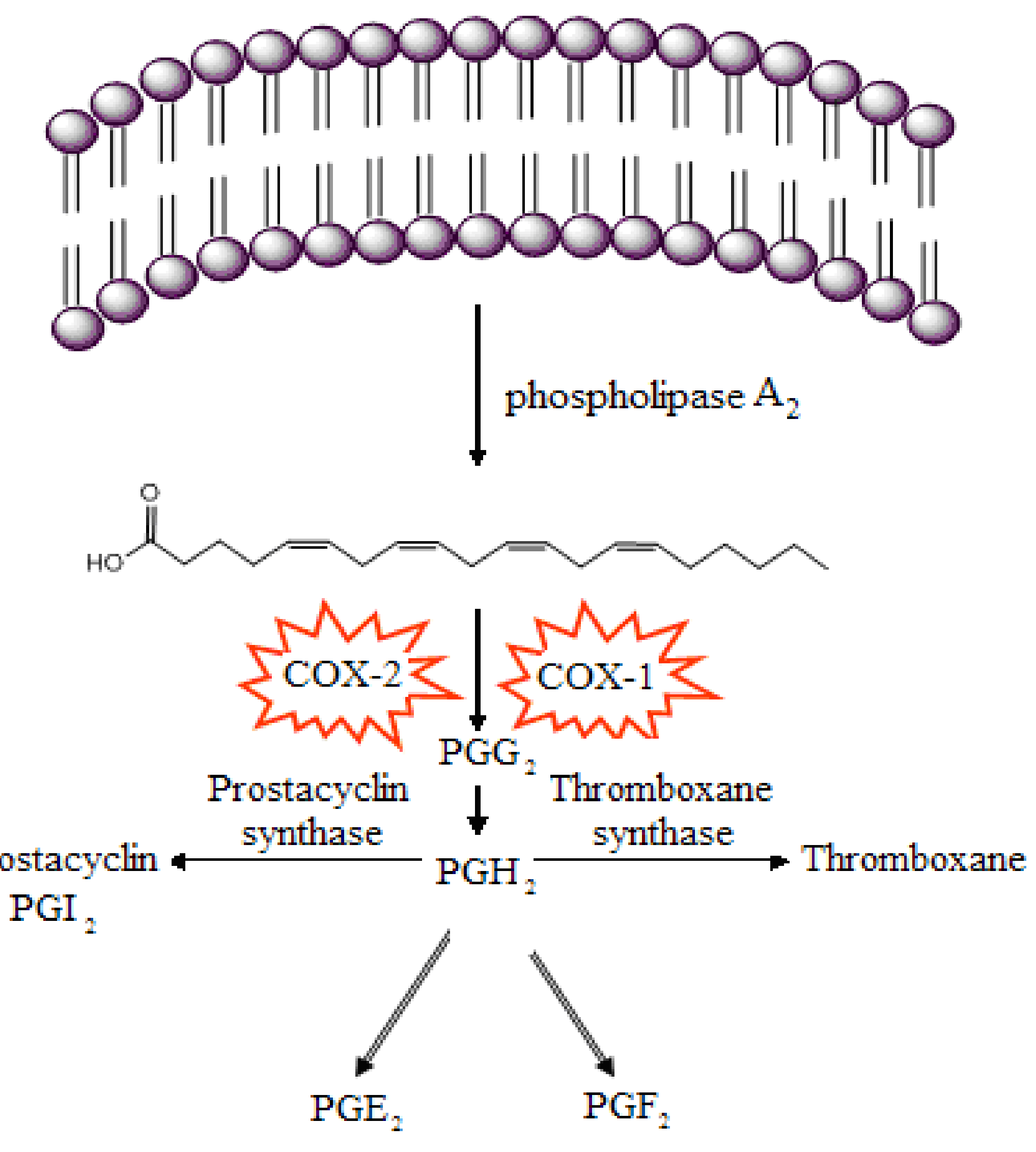

\section{INTRODUCTION}

Cyclooxygenase enzymes (COX-1 and COX-2) catalyze the biological oxidation of arachidonic acid and biosynthesis of the proinflammatory prostaglandins, which leads to pain and inflammation. Therefore, anti-inflammatory activity is achieved by inhibition of these enzymes ${ }^{1,2}$.

\section{METHODS}

This study aims to investigate the molecular docking of S-alkyl derivatives of thiosalicylic acid towards COX-1 and COX-2 enzymes. S-alkyl derivatives of thiosalicylic acid ( $\mathbf{R}=$ benzyl-, methyl-, ethyl-, propyl-, butyl-) were prepared by alkylation of thiosalicylic acid by means of the corresponding alkyl halides in alkaline water-ethanol solution (Scheme 1). All docking experiments were conducted using AutoDock Vina program. The crystal structures of the enzymes (COX-1 and COX-2), complexed with co-crystallized ligands, were obtained from Protein Data Bank (PDB ID: 1 HT5 and 4FM5).

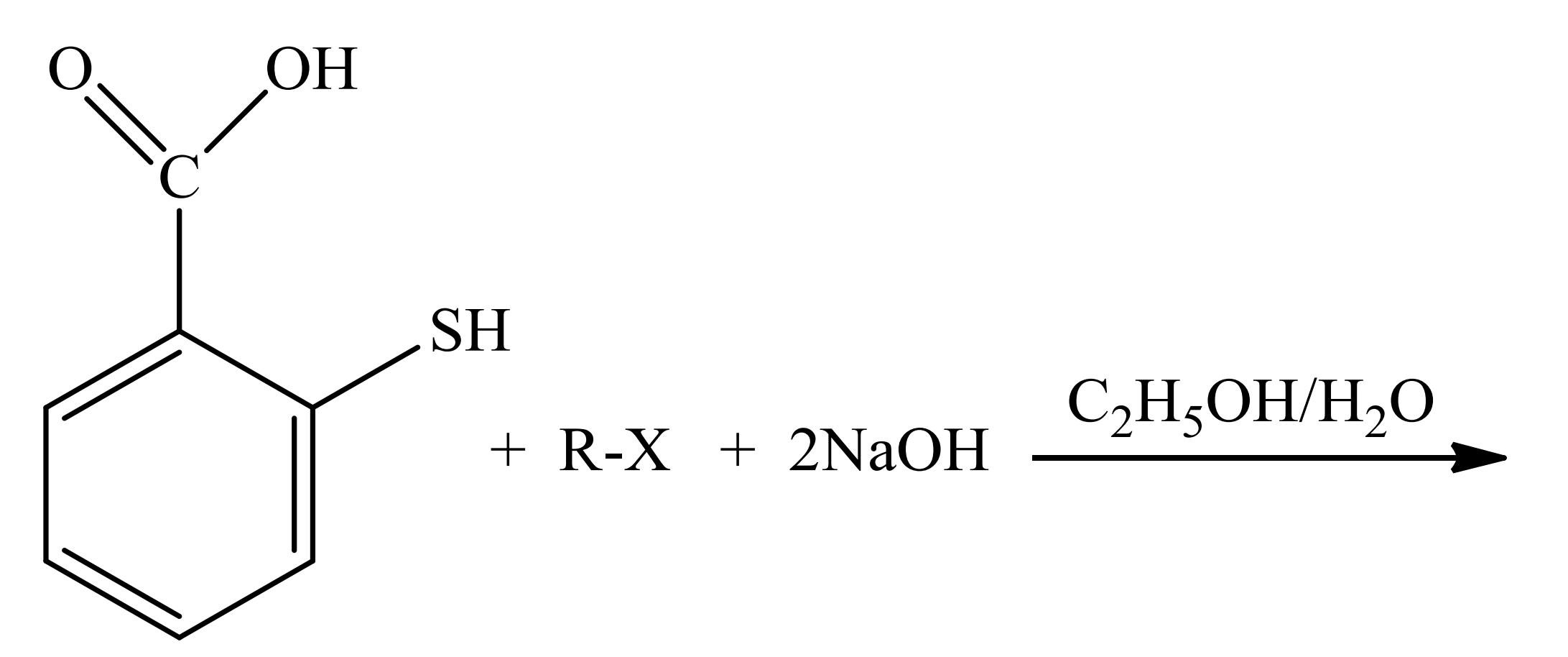<smiles>[R]Sc1ccccc1C(=O)O</smiles>

Scheme 1. The preparation of the S-alkyl derivatives of thiosalicylic acid

\section{RESULTS}

The results of molecular docking indicate that S-butyl derivatives of thiosalicylic acid shows five key binding interactions with active site of both selected target (COX-1: Ala527, Leu352, Ser530, Val349 and Tyr355; COX-2: Leu352, Ser530, Val349, Tyr355, Ala527 and Gly526 ). We evaluated the success of the pose attachment prediction of the co-crystallized ligand to the appropriate binding site in the crystal structure. We calculated docking parameter root median square deviation (RMSD) between the docked poses and the original crystallization ligand. Results showed no significant structural differences with a calculated RMSD of 1.3116 and $1.0017 \AA$ A . Binding energies of all tested compounds were similar to the corresponding co-crystallized ligands for both targets.

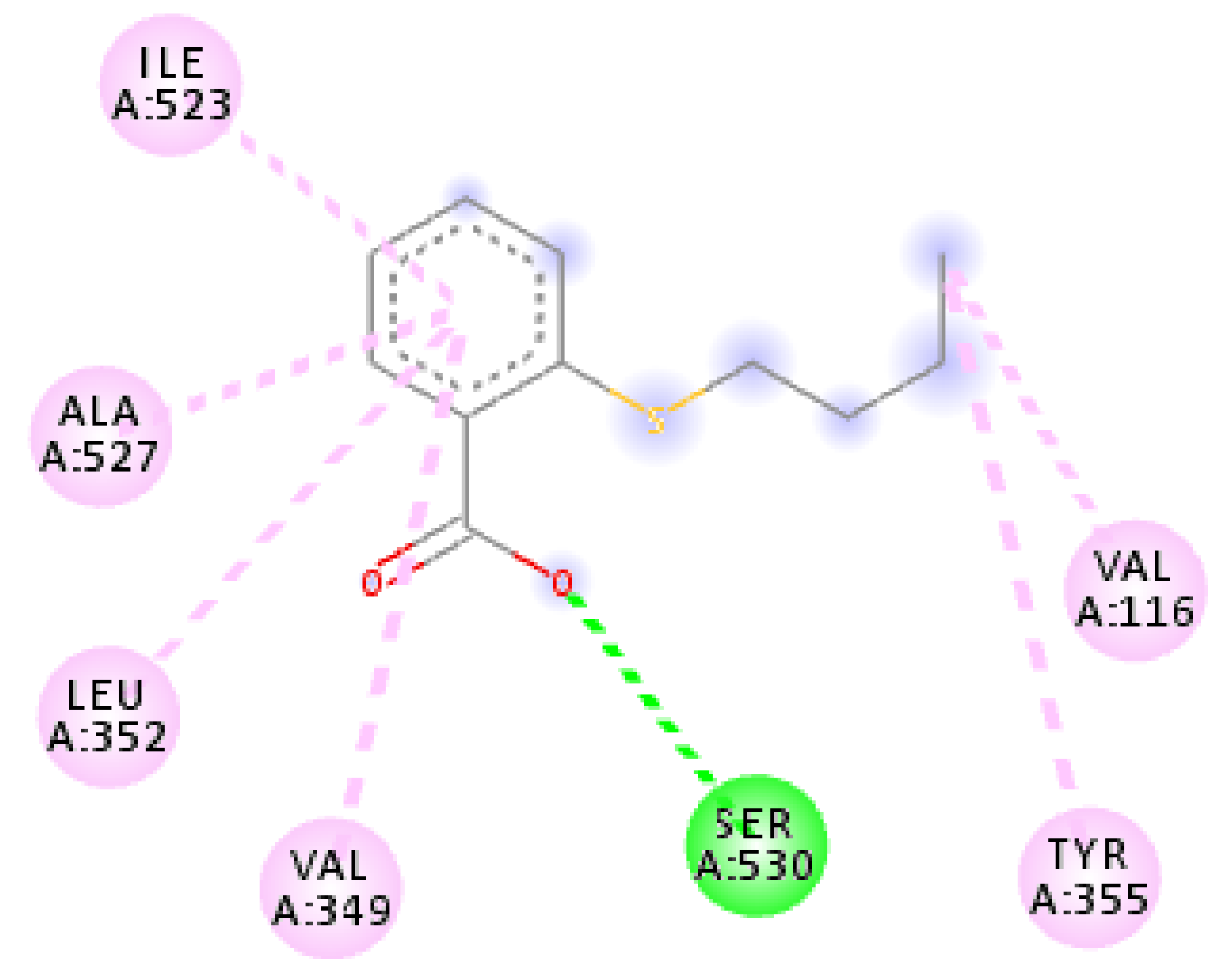

Binding mode of S-butyl derivative of thiosalicylic acid at the active site of COX-1

A)

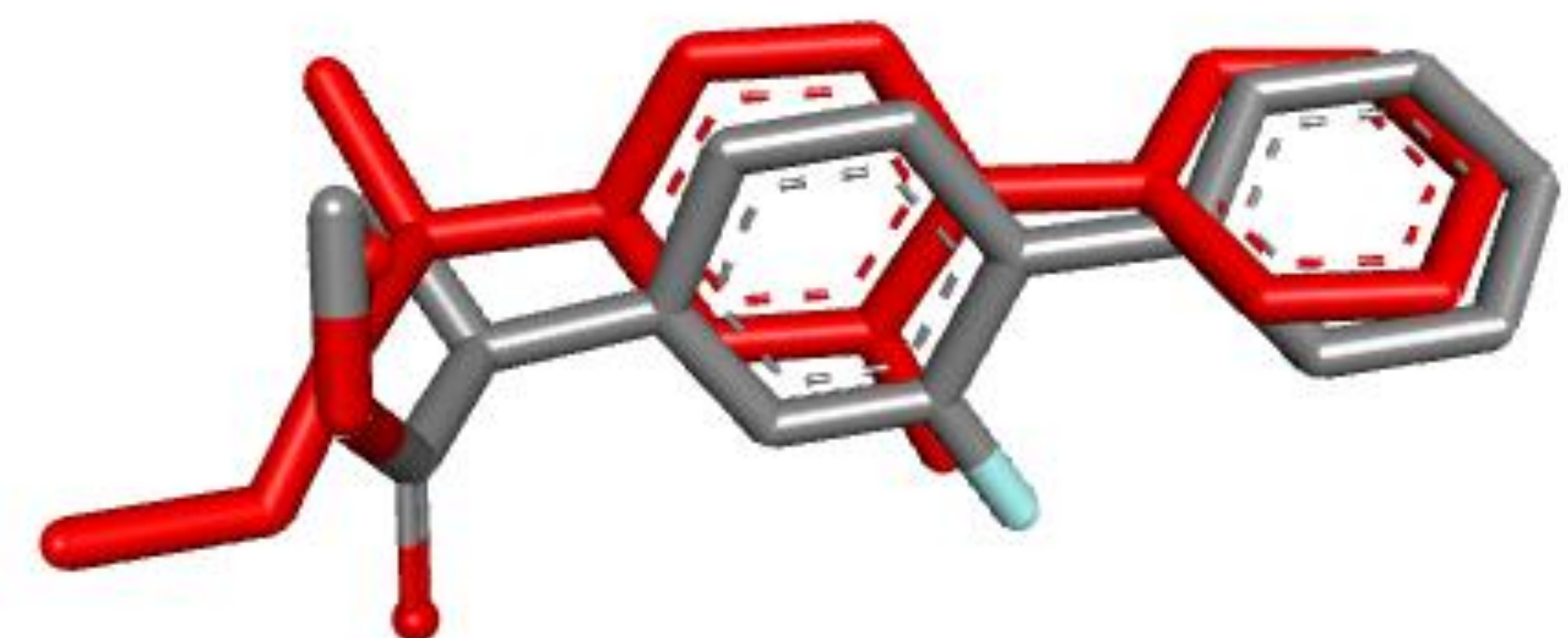

Comparison of conformation between the native ligand of the $\mathrm{X}$-ray crystal structure to the docking result (red) (A) $\mathrm{COX}-1$, (B) COX-2

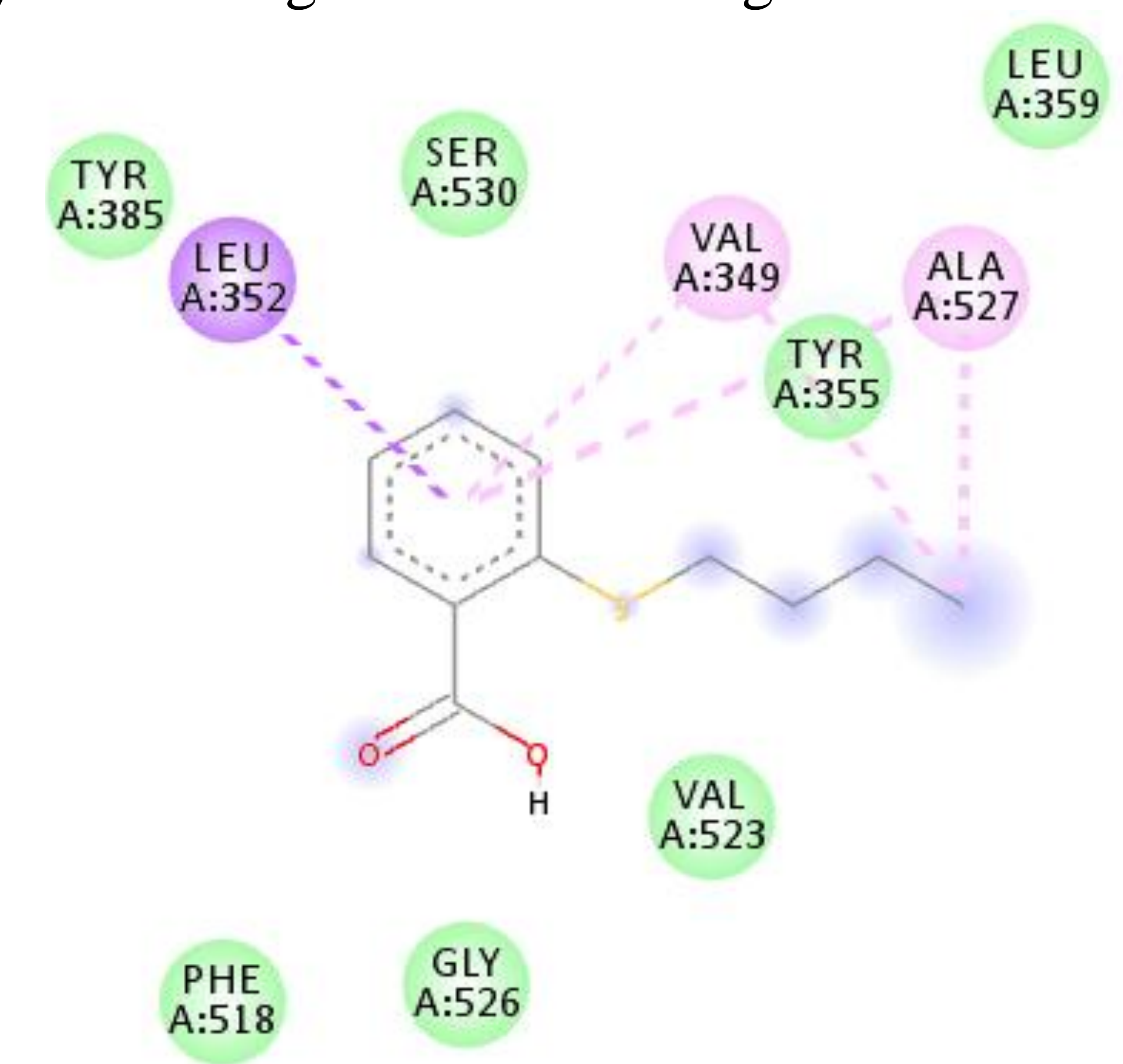

Binding mode of S-butyl derivative of thiosalicylic acid at the active site of COX-2

CONCLUSION

In conclusion, the S-alkyl derivatives of thiosalicylic acid were evaluated as inhibitors of COX-1 and COX-2 enzymes. Molecular docking studies suggested that the main interaction with active site of cyclooxygenase enzymes achieved S-butyl derivatives of thiosalicylic acid. The reason for this may be a certain structural similarity with the co-crystalized ligands (flurbiprofen and des-methylflurbiprofen).

References:

1. Fitzpatrick FA. Cyclooxygenase enzymes: regulation and function. Curr Pharm Des. 2004; 10(6): 577-88.

2. Rao P, Knaus EE. Evolution of nonsteroidal anti-inflammatory drugs (NSAIDs): cyclooxygenase (COX) inhibition and beyond. J Pharm Pharm Sci. 2008 ; 11(2): 81-110. 